\title{
An inexpensive remotely-operated video recording system for continuous behavioral
}

$7 \quad{ }^{1}$ Biology Department, University of Maryland, College Park, MD

$8 \quad{ }^{*}$ Corresponding author

$9 \quad$ E-mail: wweber@umd.edu

\section{Abstract}

Video recording technology is an important tool for studies of animal behavior because it

14 reduces observer effects and produces a record of experiments, interactions among subjects,

15 and contextual information, however it remains cost prohibitive for many researchers. Here we

16 present an inexpensive method for building a remotely-operated video recording system to

17 continuously monitor behavioral or other biological experiments. Our system employs Raspberry

18 Pi computers and cameras, open-source software, and allows for wireless networking, live-

19 streaming, and the capacity to simultaneously record from several cameras in an array. To

20 validate this system, we continuously monitored home-cage behavior of California mice

21 (Peromyscus californicus) in a laboratory setting. We captured video in both low- and bright-light

22 environments to record behaviors of this nocturnal species, and then quantified mating

23 interactions of California mouse pairs by analyzing the videos with an open-source event

24 logging software. This video recording platform offers users the flexibility to modify the

25 specifications for a range of tasks and the scalability to make research more efficient and

26 reliable to a larger population of scientists. 


\section{Introduction}

Behavior often represents an animal's most immediate and dynamic response to internal or external cues [1]. Behavioral data are, therefore, invaluable indicators of change, yet the complexity and diversity of behaviors can be challenging to quantify. Traditional sampling methods from live observations (e.g., scan sampling, focal animal sampling, one-zero sampling [2-3]) frequently results in an estimate, rather than a precise measure of behaviors, or only provides data from a subset of subjects or interactions within a social group [4]. The behavioral record is therefore often incomplete with live observational data, and the presence of an observer can influence the behavior of study subjects, thus biasing the results [5-7]. Moreover, to fully understand an animal's response, it is imperative to understand the context and the environment in which the behavior was expressed. Video recording enables researchers to review behaviors multiple times to yield a more complete dataset, reduces observational biases, and can capture contextual information.

Video recording has become a nearly ubiquitous tool for behaviorists and useful in a wide range of studies. For example, video can improve data collection in manipulative behavioral assays from studies of visual recognition in cichlids [8] and foraging in fruit flies [9], to parental care [10] and anxiety in rodents [11]. Moreover, passive monitoring approaches, such as home-cage cameras, can be used to record social behaviors, such as pair bonding in marmosets [12], or mating in whiteflies [13], guppies [14], and gerbils [15]. Outside of the lab, passive monitoring with nest box cameras have revealed unexpected behaviors including paternal care in tree swallows, even when extra-pair copulations were evident to the male [16], personality traits and social dominance in zebra finch [17], and long-term social interactions in "near natural" enclosures in house mice [4]. Continuous video recording systems provide a record of behavior and contextual information in a variety of studies from controlled laboratory assays to passive monitoring in the wild. 
With few exceptions, continuous video recording improves the rigor and repeatability in

54 behavioral studies, yet the technology remains cost prohibitive for researchers with limited

55 funding or those requiring numerous cameras for complex experimental designs. Here we

56 present an inexpensive method for building a remotely-operated video recording system using

57 open-source software that allows for wireless networking, live-streaming, and the capacity to

58 simultaneously record from several cameras to create an array. We then present validation of

59 this system, in which we built an array of modules to continuously record mating behavior in

60 California mice, Peromyscus californicus.

61

\section{Materials and Methods}

63

We constructed a video recording array using Raspberry Pi (RPi; Caldecote, Cambridge,

65 UK) modules and open-source software to continuously record multiple mating pairs of captive

66 California mice (Peromyscus californicus) across several reproductive cycles. We then

67 transferred the video files to Google Drive (Google LLC, Mountain View, CA, USA) for cloud-

68 based storage, and later scored videos using the free open-source software, BORIS (Behavioral

69 Observation Research Interactive Software [18]).

$71 \quad$ Recording module assembly

Raspberry Pi computers are very small, inexpensive computers that offer a flexible

73 platform to build a video recording system. Some base units can be purchased with cameras

74 but storage and cables must be purchased separately. Table 1 describes the basic starting

75 materials we used and ordering information. At the time of purchase, each recording module

76 cost roughly $\$ 50.00$ to build.

77 
Table 1. Basic Materials

\begin{tabular}{|l|l|}
\hline Product & Comments \\
\hline 64 Gb micro SD card & $\begin{array}{l}\text { A larger card can be purchased if the budget allows; be sure the } \\
\text { card comes with a SD card adaptor. }\end{array}$ \\
\hline Raspberry Pi Zero(W) & The "W" version must be purchased for WiFi connectivity. \\
\hline Raspberry Pi NolR Camera & \\
\hline $\begin{array}{l}\text { Pi Foundation Official Pi } \\
\text { Zero Case (Pi Zero Case) }\end{array}$ & This is the case for the assembled components. \\
\hline Micro HDMI charging cable & $\begin{array}{l}\text { A multiple port dock is advised if using multiple recording } \\
\text { devices. }\end{array}$ \\
\hline Charging dock & \\
\hline USB Hub &
\end{tabular}

80

97

To build a recording module, we first loaded all application files, including an operating system (OS), onto a micro SD card. We used $64 \mathrm{~Gb}$ micro SD cards, as roughly $8 \mathrm{~Gb}$ must be allocated to the OS and our video files averaged $30 \mathrm{~Gb}$ in size. We formatted the card as MSDOS(Fat); the "Raspbian" OS used in this application was designed to be loaded onto cards smaller than $32 \mathrm{~Gb}$, therefore the $64 \mathrm{~Gb}$ card required reformatting. Once formatted, we loaded the "Raspbian" OS (https://www.raspberrypi.org/downloads/noobs/) onto the card and then installed it into the micro SD card port of a Raspberry Pi Zero(W) board (Figure 1A).

$$
\text { Next, we attached the Raspberry Pi NolR camera to the R-Pi board (Figure 1B) and }
$$
placed the board into the Pi Zero Case (Figure 1C). This step requires extreme care, as there are locking pins (see arrows in Figure 1D) that secure the board and camera to the case, thus it was necessary to bend the case to prevent damaging the R-Pi board. Once the case was closed, the recording module was complete (Figure 1D). We next attached the recording module to a monitor, mouse and keyboard with a USB hub, and then connected the module to a power source to activate the unit. The OS automatically initializes as soon as power is connected, the activation process runs for approximately 10 minutes and then the Raspian desktop appears. 

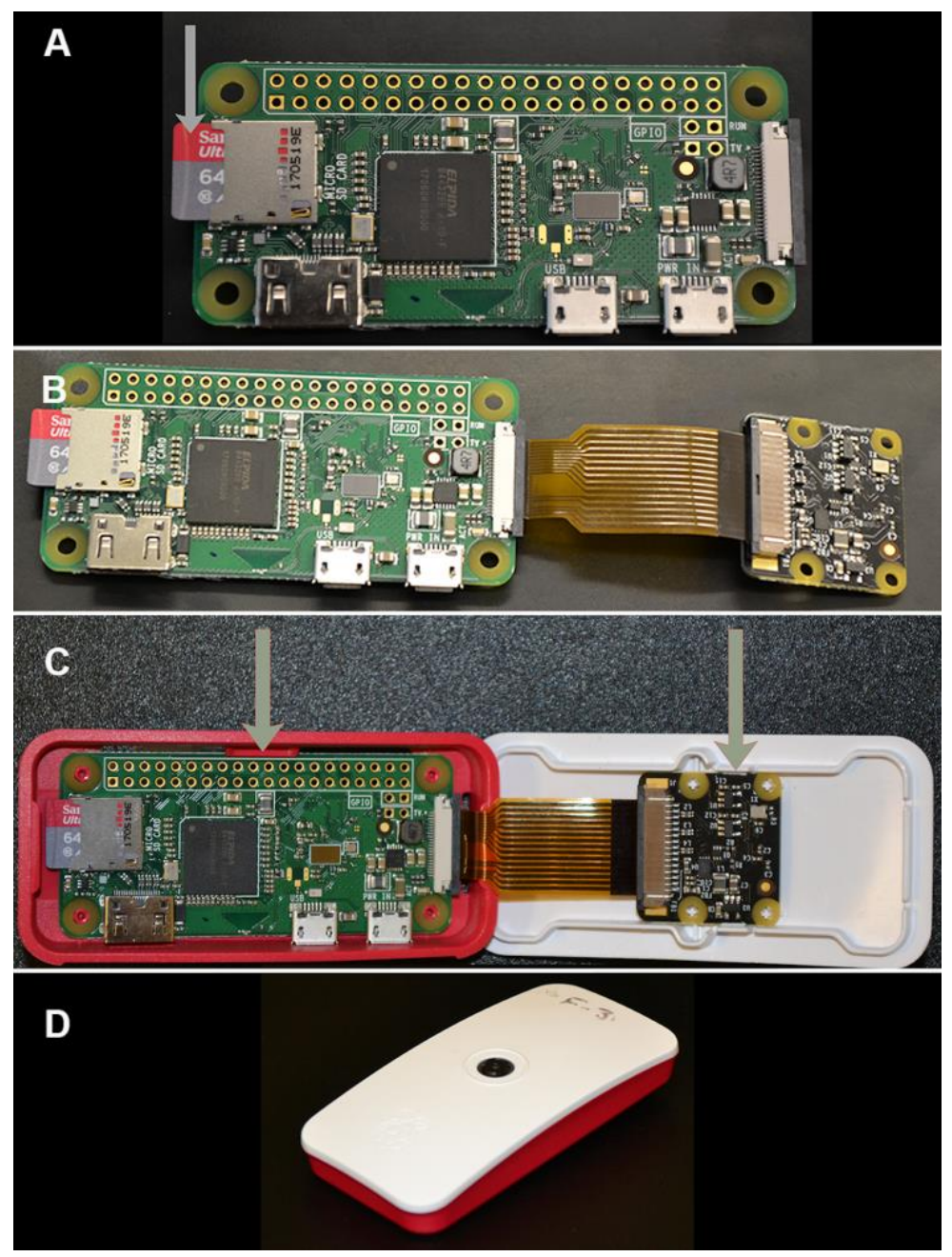

Figure 1. Assembly of a recording module. (A) MicroSD card installed into a Raspberry Pi Zero(W) board. Grey arrow indicates the location of the MicroSD card port. (B) Raspberry Pi board is attached to the camera assembly. (C) Pi Zero Case. Grey arrows indicate locking pins that the board and camera must fit underneath. (D) An assembled recording module.

\section{$\underline{\text { Recording Module Programming }}$}

On all modules, the default username is "pi" and password is "raspberry", making them susceptible to unauthorized off-network access. Therefore, we next reset the default username and password in the "Raspberry Pi Configuration Menu". Under the interface tab of that same menu, we then enabled the "Camera", selected "SSH" (allows for remote access) and "VNC" (allows for remote interfacing) options, and then rebooted the module. After reboot, we established an internet connection by hovering the cursor over the WiFi icon, selected the appropriate network, and logged in. By hovering the cursor over the WiFi icon after establishing 
113 a connection, the IP address is revealed; with this IP address we could remotely log into the

114 module using RealVNC Viewer (https://realvnc.com/en/), an open-source software.

\section{Behavioral Observations}

To assess the routine use of the video module to record social interactions, we observed mating behavior in California mice. We obtained four male and four female mice from the

119 Peromyscus Genetic Stock Center at the University of South Carolina

120 (https://www.pgsc.cas.sc.edu/), and housed them at the University of Maryland, in accordance

121 with the Institutional Animal Care and Use Committee policies (protocol number: R-Jul-18-38),

122 and in standard transparent laboratory rodent cages lined with cedar shavings. We provided food and water ad libitum and maintained all mice at $22^{\circ} \mathrm{C}$ in a $16: 8$ light-dark cycle, simulating this species' breeding season [19].

To record the mating behavior, we placed a male and a female into a cage on a camera rack (Figure 2). Camera stands can be easily purchased online or 3D printed, but we fashioned recorded behaviors during the light period with one module and used a second module to record behavior during the dark period while the room was illuminated with red lights and infrared LED lights. We found that using two modules, each set to parameters optimized for each light environment, was the most effective way for us to obtain high quality video across the entire

134 light-dark cycle, however a single camera can be programed to adjust parameters at different 135 times through the day. In our setup, the size of the files produced from using a single module 136 exceeded the capacity of the micro-SD card, and we found that adding as second module was 137 more cost effective that using a high-capacity micro-SD card. 


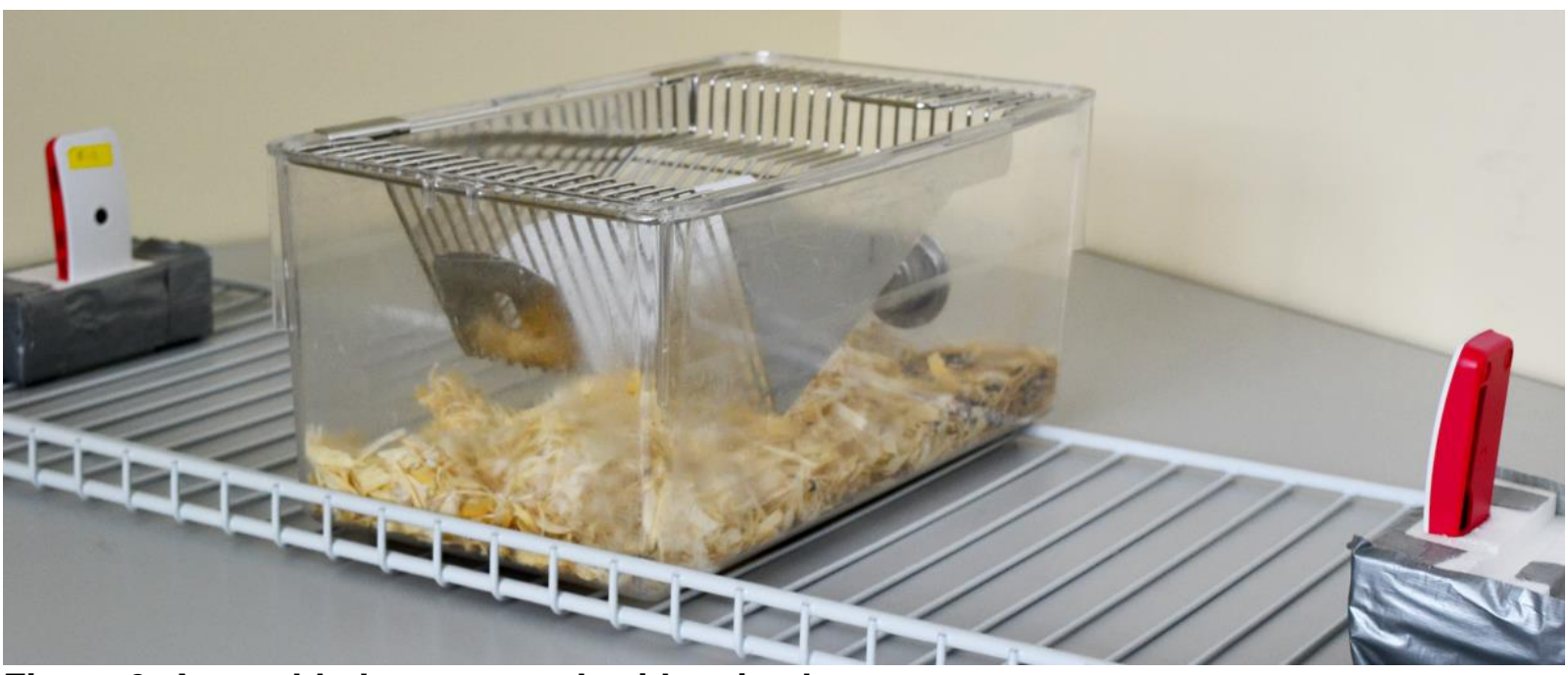

Figure 2. Assembled camera rack with animal cage.

Next, we remotely logged into the modules using a Python 3 shell (selected from the programming menu on the Raspbian desktop) and executed the camera script (Table S-1; all coding scripts described in this article can also be accessed at

https://github.com/wdavidweber/Pi-Recording-Module). To send video files directly to a Google Drive folder, we used an additional script because the software is not included in the Raspian OS. Using the terminal console in Raspbian, we uploaded the "rclone" (Nick Craig-Wood @ 2012) open-source software (Table S-2), and regularly monitored the modules and Google Drive folders. Free storage on Google Drive is limited to $15 \mathrm{~Gb}$, unless an unlimited account is available to you, therefore, files may need to be downloaded and transferred prior to filling the storage. To score the behaviors in BORIS, we converted the .h264 format files (the default Raspbian filetype) into ".mp4" format using the "ffmpeg" package, available in the Raspbian OS (the code for this is the last line of script in Table S-1). We performed the file conversion using a High-Performance Computing Cluster at the University of Maryland because our video files were too large and numerous to be converted on the modules. In most situations, this can be done on the module itself, or the conversion can be performed on any workstation. When necessary, we live-streamed footage with a YouTube account (https:www.youtube.com/) by 
loading "Docker" onto the module (Table S-3). With this method, video recording and livestreaming cannot be accomplished simultaneously.

To quantify mating behavior, we scored videos using the open-source software, BORIS, and developed an ethogram based on the work of Dewsbury [20]. We recorded the frequency of mounts, intromissions, and ejaculations, as well as intromission latency (the time between the 163 start of a reproductive bout and the first intromission), ejaculation latency (the time between the

164 first intromission and the subsequent ejaculation), post-ejaculatory interval (the time between an 165 ejaculation and the beginning of the next copulatory bout), reproductive event (the total duration 166 of all mating events during a female's single reproductive period, beginning with the first 167 mounting event and concluding with the last ejaculation), copulatory bout (the duration between 168 the first mounting event and the first ejaculation).

\section{Results}

We continuously recorded home-cage behavior for three weeks from four pairs of

173 California mice. In total, we collected 2016 hours of video, which resulted in roughly 2 TB of

174 data. Recording one hour of footage during the light period (Figure 3A-B) under white lights, at a 175 resolution of $1280 \times 768$, a framerate of 15 frames per second, and a brightness value of 70 , 176 resulted in recordings that were $440 \mathrm{Mb}$ to $500 \mathrm{Mb}$ in size. Recording one hour of footage during 177 the dark period (under room red lights and LED infrared lights mounted above the cage; Figure 178 3C-D) at a resolution of $1280 \times 768$, a framerate of 15 frames per second, and a brightness 179 value of 80 , resulted in recordings of $1.5 \mathrm{~Gb}$ to $2 \mathrm{~Gb}$ in size. This difference in brightness 180 variables was the result of room illumination, there is more illumination in the room under white 181 light than while under red light. By reducing the parameters for resolution, framerate, or 182 brightness small files are generated, but the footage is less resolved. 



Figure 3. Recording module array and output. (A) The array during the light period with room illuminated by white lights, note the infrared LED lights over the cages (not needed for bright light conditions, shown here as an example). (B) Still of a video obtained during the light period, one animal as a spot shaven on his back for identification purposes. (C) The same array in (A) but the image is taken from the opposite side while during the dark period under room red lights and infared LED lights mounted above cages (not shown here). (D) Still image from a video obtained during the dark period.

We scored mating behavior from the one pair that successful produced a litter after the

mating trial. The active mating period spanned a total of 5 hours, 24 minutes, and 3 seconds of

194 footage, all of which occurred during the dark period of the photocycle and included two

copulatory bouts. The first mating bout spanned 4 minutes, 15 seconds, and included 8 mounts,

3 intromissions and a single ejaculation; the second bout spanned 2 minutes, 45 seconds and

197 included 6 mounts, 5 intromissions and a single ejaculation. We found that the mean

198 intromission latency was 1 minute, 49 seconds and the mean ejaculation latency was 1 minute

19942 seconds. The two copulatory bouts were separated by a 36 minutes, 39 second refractory

200 period. 


\section{Discussion}

Here we describe a remotely-operated video recording system to monitor animal

204 behavior using wireless networking. This tool permits user-determined specifications, including

205 live-streaming and multiplexing, and has the capacity to simultaneously record and upload data

206 files for storage. Moreover, this system allows for continuous behavioral monitoring on a flexible

207 timescale. The cost of the recording module that we describe is less or comparable to some

208 pre-assembled commercial surveillance cameras, which like the RPi recording module, often

209 use SD cards to store footage and may provide cloud storage for a fee with restricted

210 allocations. However the system described here offers a wider range of low-cost modifications

211 to suit a variety of research environments by incorporating open-source software add-ons.

212 For validation purposes, we used the video capture system we report here to record, and

213 subsequently quantify, mating behavior in the California mouse. Once we positioned the

214 cameras in place in our animal facility, we controlled the modules from our laboratory located in

215 another building. This design eliminated possible observer effects on the animals' behavior,

216 provided contextual information on the animals' environment, and allowed for off-hours

217 monitoring of the animal housing space. Furthermore, with the addition of infrared LED lights,

218 we were able to record mating behavior of this nocturnal species without interruption or

219 disturbance.

In the research setting, our video recording system can be applied to tasks beyond

221 animal behavior experiments. For example, this system could be used to remotely monitor

222 chemical reactions or other assays in which timing is uncertain, or if the modules are housed

223 within an incubator, to monitor bacterial or embryo culture. The flexible platform, scalability and

224 low cost of our "do it yourself" video recording system is a powerful lab tool to make research

225 more efficient and reliable to a larger population of scientists. 


\section{Acknowledgements}

228 We thank Andrés Bendesky, whose use of Raspberry Pi cameras to record parental care

229 behavior in Peromyscus inspired us to build and report on the system presented here. We also

230 thank Irene Lui for her computer programming guidance, Erica Glasper for the experimental

231 animals, and R. Zaak Walton, whose thoughtful discussion and advice were essential to this

232 project.

\section{References}

235 1. Bretman A, Gage M, Chapman T. Quick-change artists: male plastic behavioural

236 responses to rivals. Trends Ecol Evol. 2011;26(9):467-73.

2372 2. Altmann J. Observational Study of Behavior : Sampling Methods. Behaviour [Internet].

238 1974;49(3/4):227-67.

239 3. Martin P, Bateson P. Measuring behaviour: An introductory guide. 2nd, editor.

240 Cambridge, EN, UK: Cambridge University Press; 1995.

241 4. Weissbrod A, Shapiro A, Vasserman G, Edry L, Dayan M, Yitzhaky A, et al. Automated long-term tracking and social behavioural phenotyping of animal colonies within a seminatural environment. Nat Commun. 2013;4(May):1-10.

5. Wilson S. The use of ethnographic techniques in educational eesearch. Rev Educ Res. 1977;47(2):245-65.

6. Monahan T, Fisher J. Benefits of "observer effects": Lessons from the field. Qual Res. 2010;10(3):357-76.

7. Herbert R, James F. The Hawthorne experiments: first statistical interpretation. Am Sociol Rev. 1978;43(5):623-43.

8. Escobar-Camacho D, Marshall J, Carleton K. Behavioral color vision in a cichlid fish: Metriaclima benetos. J Exp Biol [Internet]. 2017;220(16):2887-99.

10. Bendesky A, Kwon YM, Lassance JM, Lewarch CL, Yao S, Peterson BK, et al. The

9. Jang EB, Light DM. Behavioral responses of female oriental fruit flies to the odor of papayas at three ripeness stages in a laboratory flight tunnel (Diptera: Tephritidae). $\mathrm{J}$

257 11. Leuner B, Glasper ER, Gould E. Sexual experience promotes adult neurogenesis in the 
hippocampus despite an initial elevation in stress hormones. PLoS One. 2010;5(7).

12. Gerber P, Schnell C, Gusti A. Behavioral and cardiophysiological responses of Common Marmosets (Callithrixjacchus) to social and environmental changes. Primates. 2002;43(July):201-16.

13. Ruan YM, Luan JB, Zang LS, Liu SS. Observing and recording copulation events of whiteflies on plants using a video camera. Entomol Exp Appl. 2007;124(2):229-33.

14. Pilastro A, Mandelli M, Gasparini C, Dadda M, Bisazza A. Copulation duration, insemination efficiency and male attractiveness in guppies. Anim Behav. 2007;74(2):3218.

15. Prates EJ, Guerra RF. Parental care and sexual interactions in Mongolian gerbils (Meriones unguiculatus) during the postpartum estrus. Behav Processes. 2005;70(2):104-12.

16. Whittingham L, Dunn P, Robertson R. Confidence of paternity and male parental care: an experimental study in tree swallows. Anim Behav. 1993;139-47.

17. Morgan D, Auclair Y, Cezilly F. Personality predicts social dominance in female zebre finches, Taeiopygia guttata, in a feeding context. Anim Behav. 2011;81(1):219-24. 


\section{Supplemental Material}

290 Table S-1. Camera operation and recording script

\begin{tabular}{|l|l|}
\hline Line & Python Script \\
\cline { 1 - 1 } 2 & from picamera import PiCamera \\
from time import sleep \\
import os
\end{tabular}

Explanation: (1) This line activates the software for the camera. (2) This line tells the unit to use

292 the sleep software for timing. (3) This command allows the OS to operate in the python shell. (4)

293 Skip this line. (5) This line instructs the camera to execute the following script. (6) Skip this line.

294 (7) This sets the resolution for the recording. (8) This sets the framerate for the recording. (9)

295 This sets the brightness for the recording. (10) This command begins camera operations at the script execution. (11) This command begins recording at the execution, and places files where directed [e.g., (")]. (12) This command sets the recording time (e.g., 61200 seconds). (13) Skip this line. (14) This line instructs the R-Pi to convert the recording into .mp4 format. (15) This command uploads the recording onto the specified Google Drive folder. (16) This removes the .h264 file that was used to make the .mp4 video. 
Table S-2. Rclone installation and GDrive connection script

\begin{tabular}{|c|c|}
\hline Line & Unix Script \\
\hline 1 & \multirow{14}{*}{$\begin{array}{l}\text { curl -L http://raw.github.com/pageauc/pi-timolo/master/source/pi-timolo-install.sh | } \\
\text { bash } \\
\text { rclone config } \\
\mathrm{n} \\
\text { picam } \\
\text { storage> } 12 \\
\text { client id }> \\
\text { client_secret> } \\
\text { scope }>1 \\
\text { root_folder_id }> \\
\text { service_account_file> } \\
\text { y/n }>\text { n } \\
\text { y/n }>\text { n } \\
y / n>n \\
y / e / d>y\end{array}$} \\
\hline 2 & \\
\hline 3 & \\
\hline 4 & \\
\hline 5 & \\
\hline 6 & \\
\hline 7 & \\
\hline 8 & \\
\hline 9 & \\
\hline 10 & \\
\hline 11 & \\
\hline 12 & \\
\hline 13 & \\
\hline 14 & \\
\hline
\end{tabular}

Explanation: (1) This script uploads the rclone software onto the R-Pi, the script will take several

313 minutes to run. (2) This command opens the rclone configurations. (3) The next prompt will ask

314 you to make a new or edit a remote, "n" indicates new. (4) The next prompt will ask for a name

315 of the new remote, which can be anything but must match the code in Table 2, line 15 (e.g.,

316 picam). (5) There are many virtual cloud storage options with the rclone software, this prompt will ask you to select the one from the list that appears, in our case that was "12" for GDrive. (6)

Leave this prompt blank. (7) Leave this prompt blank. (8) This prompt allows you to select permissions of the connection. As long as a secured network is used, full access or " 1 " is the best setting. (9) Leave this prompt blank. (10) Leave this prompt blank. (11) In this prompt, we selected " $n$ " to not use advanced settings. (12) In this prompt, we selected " $n$ " for auto configuration. The result of this is a URL that must be copied into a web browser that is logged copied and pasted into the next prompt. (13) This prompt asks if the account is a Team Drive, for which we selected "n" for no. (14) In the final prompt, there should be a list of remotes where the new remote should appear, it will ask if everything looks correct, we selected " $y$ " for yes. 
Table S-3. Docker script for live-streaming

\begin{tabular}{|c|c|}
\hline Line & Unix Script \\
\hline 1 & \multirow{5}{*}{$\begin{array}{l}\text { curl -sSL https://get.docker.com | sh } \\
\text { sudo usermod pi -aG docker } \\
\text { reboot } \\
\text { docker pull alesellis2/streaming:07-05-2018 } \\
\text { docker run --privileged --name XXX -ti alexellis2/streaming:07-05-2018 XX-XXX-XXXX }\end{array}$} \\
\hline 2 & \\
\hline 3 & \\
\hline 4 & \\
\hline 5 & \\
\hline
\end{tabular}
Explanation: (1) This script uploads the docker software onto the R-Pi, the script will take

338 several minutes to run. (2) This script initiates the docker settings in the R-Pi and will again take 339 several minutes to run. (3) This command reboots the R-Pi. (4) Once the terminal is opened 340 again, this command updates the docker structure in the R-Pi. At this point the R-Pi is ready to 341 live stream. (5) This script activate the live-streaming. The first series of XXX was a unique 342 name for the particular stream, the second set of XX-XXX-XXXX represents the YouTube key 343 associated with the account. 